\title{
Building a reference transcriptome for Juniperus squamata (Cupressaceae) based on single-molecule real-time sequencing
}

\author{
Yufei Wang ${ }^{\dagger}$, Siyu Xie ${ }^{\dagger}$, Jialiang Li, Jieshi Tang, Tsam Ju and Kangshan Mao ${ }^{*}$ (i)
}

\begin{abstract}
Objectives: Cupressaceae is the second largest family of coniferous trees (Coniferopsida) with important economic and ecological values. However, like other conifers, the members of Cupressaceae have extremely large genome $(>8$ gigabytes), which limited the researches of these taxa. A high-quality transcriptome is an important resource for gene discovery and annotation for non-model organisms.

Data description: Juniperus squamata, a tetraploid species which is widely distributed in Asian mountains, represents the largest genus, Juniperus, in Cupressaceae. Single-molecule real-time sequencing was used to obtain full-length transcriptome of Juniperus squamata. The full-length transcriptome was corrected with Illumina RNA-seq data from the same individual. A total of 47,860 non-redundant full-length transcripts, N50 of which was 2839, were obtained. A total of 57,393 simple sequence repeats were identified and 268,854 open reading frames were predicted for Juniperus squamata. A BLAST alignment against non-redundant protein database was conducted and 10,818 sequences were annotated in Gene Ontology database. InterPro analysis shows that 30,403 sequences have been functionally characterized against its member database. This data presents the first comprehensive transcriptome characterization of Juniperus species, and provides an important reference for researches on the genomics and evolutionary history of Cupressaceae plants and conifers in the future.
\end{abstract}

Keywords: Juniperus squamata, Single-molecule real-time sequencing, Simple sequence repeats, Gene ontology annotation

\section{Objective}

Compared with other plant groups, the genome analysis of coniferous species lags behind because of their larger genome [1,2]. At present, only a few genome-wide datasets are available, such as Sequoiadendron gigantea, Pinus taeda L. and Picea abies [3-5]. Whole genome sequencing of conifers is prohibitively expensive for large genome sizes, and it also produces datasets which

\footnotetext{
*Correspondence: maokangshan@163.com; maokangshan@scu.edu.cn †Yufei Wang and Siyu Xie contributed equally to this work. Key Laboratory of Bio-Resource and Eco-Environment of Ministry of Education, College of Life Sciences, State Key Laboratory of Hydraulics and Mountain River Engineering, Sichuan University, Chengdu 610064, China
}

are inconvenient to analyze. In contrast, analyses on the dataset produced by transcriptome sequencing is much easier, and it is a convenient and cost-effective method for sequencing coding sequences of complex genomes.

Juniperus squamata is an evergreen shrub of the family Cupressaceae reaching $1-3 \mathrm{~m}$ tall, with brownish-gray bark [6]. It is found in mountains from southwestern China to northeastern Afghanistan, with separate populations east to Fujian and north to western Gansu in China [7]. This tetraploid species is not only of great value to gardening but also of enormous ecological values in subalpine and alpine shrubland ecosystems in Asian mountains. However, very limited genomic information is available for this species. Hence the objective 
of this work is to generate full-length transcriptome sequences for Juniperus squamata. Considering the importance of simple sequence repeats (SSRs) to plant population genetic analysis, we also developed SSRs for this species $[8,9]$. To functionally characterize the fulllength transcriptome, open reading frame (ORF) prediction and Gene Ontology (GO) annotation analysis were performed [10]. To functionally analyze the protein, the final isoforms were searched against InterPro's predictive models [11]. The full-length transcriptome data set of Juniperus squamata can provide an important reference for its downstream analysis, such as genomic basis of environmental adaptation and genome evolution of Cupressaceae and even conifers.

\section{Data description}

Fresh leaves, stems, and strobiles of one Juniperus squamata individual were collected from Kangding, Sichuan Province, China. For each tissue, the short paired reads were sequenced by Illumina platform. We also mixed the samples of each tissue and generated the long reads by the PacBio Sequel platform. Total RNA of the samples was isolated using the Plant RNA kit (Omega bio-Tech., USA) and then treated with RNase-free DNase I (NEB) to remove DNA. RNA degradation and contamination were monitored on $1 \%$ agarose gels and RNA purity was checked using the NanoPhotometer ${ }^{\circledR}$ spectrophotometer (IMPLEN, CA, USA). RNA concentration was measured using Qubit ${ }^{\circledR}$ RNA Assay Kit in Qubit ${ }^{\circledR} 2.0$ Fluorometer (Life Technologies, CA, USA). RNA integrity was assessed using the Bioanalyzer 2100 system (Agilent Technologies, CA, USA). The Single-molecule real-time (SMRT) bell library was constructed with the Pacific Biosciences DNA Template Prep Kit 2.0 and SMRT sequencing was then performed on the Pacific Bioscience Sequel System. The sample used for Illumina sequencing was harvested using the same methods. The library was constructed using Illumina HiSeq X Ten. Adapter clipping and quality filtering of the Illumina raw reads was done using Trimmomatic version 0.36 [12]. Based on the quality check, the last two base pairs from each read were removed to minimize the overall sequencing error.

The raw full-length transcriptome sequencing data of samples were processed using the SMRT link version 4.0 software (https://www.pacb.com/support/ softwaredownloads). Subread BAM files were generate from raw reads, parameters: -minLength 200, - minReadScore 0.75. Circular consensus sequence (CCS) was generated from subread BAM files, parameters: -min_length 50,-max_drop_fraction 0.8 , - no_polish TRUE, -min_zscore $-9999.0,-$ min_passes $2,-$ min $_{-}$ predicted_accuracy $0.8,-$ max_length 15,000 . CCS
BAM files were output, which were then classified into Full-Length non-chimeric (FLNC) and non-full length (NFL) fasta files by examining the $5^{\prime}$ and $3^{\prime}$ adapters and the poly(A) tail. Iterative Clustering and Error Correction (ICE) algorithm was utilized to cluster FLNC fasta files to obtain cluster consensus. Quiver from SMRT link (parameters: -hq_uiver_min_accuracy 0.99, -bin_by_primer false, -bin_size_kb 1, -qv_trim_5p $100,-$ qv_trim_3p 30) were then utilized to polish cluster consensus sequence with NFL fasta files to obtain polished consensus sequence.

To obtain high quality corrected consensus sequence, additional nucleotide errors in polished consensus sequence were corrected using the Illumina RNA-seq data obtained from the same individual with the software LoRDEC version 0.7 [13] (parameters: -k 23 -s 3). Any redundancy in corrected consensus sequence was removed by CD-HIT version 4.6.1 [14] (parameters: -c 0.95 -T 6 -G 0 - aL 0.00 -aS 0.99 -AS 30) to obtain final a set of unique transcript isoforms. Benchmarking universal single-copy orthologs (BUSCO) version 3 was used to assess the quality of final transcript isoforms [15]. The summary statistics and length distributions of the PacBio SMART sequencing are shown in Data file 1 (Table S1 and Fig. S1). The results of BUSCO are shown in Data file 1 (Table S2). All three data sets obtained and their NCBI GenBank Accession numbers are listed in Table 1 (Data set 1, Data set 2, and Data set 3).

MISA version 1.0 was employed to identify SSRs from final unique transcript isoforms of Juniperus squamata [16] (parameters: definition (unit_size, min repeats): 1-10 2-6 3-5 4-5 5-5 6-5, interruptions (max_difference_betw-een_2_SSRs): 100). Finally, 57, 393 SSRs were identified which were containing in 42, 273 sequences. The details of SSRs of Juniperus squamata, including primer sequences, SSR type, annealing temperature, product size etc., are shown in Data file 2. TransDecoder version 5.5.0 (https://github.com/ TransDecoder/TransDecoder) was employed to identify ORF within the transcripts of Juniperus squamata. The results of ORF prediction are shown in Data file 3.

DIAMOND version 2.0.9.147 was used to align the final unique transcript isoforms against non-redundant protein database with a significance threshold of $\mathrm{E} \leq 10^{-5}$ [17]. A custom python (https://www.python. org/) script was used to carry out GO annotation (available at https://github.com/shanzha09/GO-annot ation.git). InterProScan version 5.52-86.0 was used to search the final isoforms against interPro database [18]. The results of BLASTX alignment, GO annotation, and interPro analysis are shown in Data file 4, Data file 5, and Data file 6, respectively. 
Table 1 Overview of data files/sets

\begin{tabular}{|c|c|c|c|}
\hline Label & Name of data file/data set & File types (file extension) & Data repository and identifier (DOI or accession number) \\
\hline Data file 1 & Summary and assessment of the data set & MS Word file(.docx) & Figshare (https://doi.org/10.6084/m9.figshare.14572125) [19] \\
\hline Data file 2 & SSRs of Juniperus squamata & MS Excel file(.csv) & Figshare (https://doi.org/10.6084/m9.figshare.14572098) [20] \\
\hline Datafile 3 & Longest open reading frame prediction & gff3(.gff3) & Figshare (https://doi.org/10.6084/m9.figshare.16870147) [21] \\
\hline Data file 4 & Alignment results of Juniperus squamata & text(.tab) & Figshare (https://doi.org/10.6084/m9.figshare.16870333) [22] \\
\hline Data file 5 & Go annotation results of Juniperus squamata & text(.annotation) & Figshare (https://doi.org/10.6084/m9.figshare.16870401) [23] \\
\hline Data file 6 & InterPro analysis results of Juniperus squamata & gff3(.gff3) & Figshare (https://doi.org/10.6084/m9.figshare.16912615) [24] \\
\hline Data set 1 & js.fasta.gz & fastq (fastq.gz) & NCBI(SRR13966305) [25] \\
\hline Data set 2 & juniperus_squamata_final.fasta.gz & fastq (.fastq.gz) & NCBI(SRR13993906) [26] \\
\hline Data set 3 & $\begin{array}{l}\text { Juniperus_squamata_final_unique_transcript_ } \\
\text { isoforms.fastq.gz }\end{array}$ & fastq (.fastq.gz) & NCBI(SRR14000623) [27] \\
\hline
\end{tabular}

\section{Limitations}

There is a shortcoming that we only collected one sample for single-molecule real-time sequencing of transcriptome.

\section{Abbreviations}

BUSCO: Benchmarking universal single-copy orthologs; CCS: Circular consensus sequence; FLNC: Full-length non-chimeric; ICE: Iterative Clustering for Error Correction; NFL: Non-full length; ROI: Reads of insert; SMRT: Single-molecule real-time; SSRs: Simple sequence repeats.

\section{Acknowledgements}

The authors acknowledge financial support by the National Natural Science Foundation of China (grant number U20A2080, 31622015) and Sichuan University (Fundamental Research Funds for the Central Universities, SCU2021D006, SCU2020D003).

\section{Authors' contributions}

SX, JL, YJ and KM collected the samples, YW and SX analyzed the data, YW wrote the note. JT, JL, TJ and KM revised the manuscript, KM conceived and designed the program. All authors have read and approved the manuscript.

\section{Funding}

The project was supported by National Natural Science Foundation of China (grant number U20A2080, 31622015) and Sichuan University (Fundamental Research Funds for the Central Universities, SCU2021D006, SCU2020D003). The funding bodies played no role in the design of the study and collection, analysis, and interpretation of data and in writing the manuscript.

\section{Availability of data and materials}

The data described in this Data note can be freely and openly accessed on NCBI under SRR13966305, SRR13993906 and SRR14000623. Please see Table 1 and references Data file 1,2,3,4,5\&6 and Data set 1,2 \& 3 for details and links to the data.

\section{Declarations}

Ethics approval and consent to participate

Not applicable.

\section{Consent for publication}

Not applicable.

\section{Competing interests}

The authors declare no competing interests.
Received: 12 April 2021 Accepted: 19 November 2021

Published online: 05 December 2021

\section{References}

1. De La Torre AR, Birol I, Bousquet J, Ingvarsson PK, Jansson S, Jones SJM, et al. Insights into conifer giga-genomes. Plant Physiol. 2014;166(4):172432. https://doi.org/10.1104/pp.114.248708.

2. Prunier J, Verta JP, MacKay JJ. Conifer genomics and adaptation: at the crossroads of genetic diversity and genome function. New Phytol. 2016;209(1):44-62. https://doi.org/10.1111/nph.13565.

3. Lu MM, Krutovsky KV, Loopstra CA. Predicting adaptive genetic variation of loblolly pine (Pinus taeda L.) populations under projected future climates based on multivariate models. J Hered. 2019;110(7):857-65. https://doi.org/10.1093/jhered/esz065.

4. Scott AD, Zimin AV, Puiu D, Workman R, Britton M, Zaman S, et al. A reference genome sequence for Giant Sequoia. G3: Genes|Genomes|Genetics. 2020;10(11):3907-19. https://doi.org/10.1534/g3.120.401612.

5. Nystedt B, Street NR, Wetterbom A, Zuccolo A, Lin Y-C, Scofield DG, et al. The Norway spruce genome sequence and conifer genome evolution. Nature. 2013;497(7451):579-84. https://doi.org/10.1038/nature12211.

6. Wu Z, Peter HR, Hong D. CUPRESSACEAE. In: Fu L, Yu Y, Aljos F, editors. Flora of China, vol. 4. Saint Louis: Missouri Botanical Garden Press; 1999. p. 62-77.

7. Adams RP. Junipers of the world: the genus Juniperus. 4th ed. Bloomington: Trafford Publishing Company; 2014.

8. Vieira MLC, Santini L, Diniz AL, Munhoz CF. Microsatellite markers: what they mean and why they are so useful. Genet Mol Biol. 2016;39:312-28. https://doi.org/10.1590/1678-4685-GMB-2016-0027.

9. Zhang Q, Li J, Zhao Y, Korban SS, Han Y. Evaluation of genetic diversity in Chinese wild apple species along with apple cultivars using SSR markers. Plant Mol Biol Report. 2012;30(3):539-46. https://doi.org/10.1007/ s11105-011-0366-6.

10. Consortium GO. The gene ontology (GO) database and informatics resource. Nucleic Acids Res. 2004;32(suppl_1):D258-61. https://doi.org/ 10.1093/nar/gkh036.

11. Finn RD, Attwood TK, Babbitt PC, Bateman A, Bork P, Bridge AJ, et al. InterPro in 2017-beyond protein family and domain annotations. Nucleic Acids Res. 2017;45(D1):D190-9. https://doi.org/10.1093/nar/gkw1107.

12. Bolger AM, Lohse M, Usadel B. Trimmomatic: a flexible trimmer for Illumina sequence data. Bioinformatics. 2014;30(15):2114-20. https://doi. org/10.1093/bioinformatics/btu170.

13. Salmela L, Rivals E. LoRDEC: accurate and efficient long read error correction. Bioinformatics. 2014;30(24):3506-14. https://doi.org/10.1093/bioin formatics/btu538. 
14. LiW, Godzik A. Cd-hit: a fast program for clustering and comparing large sets of protein or nucleotide sequences. Bioinformatics. 2006;22(13):1658-9. https://doi.org/10.1093/bioinformatics/btl158.

15. Simão FA, Waterhouse RM, loannidis P, Kriventseva EV, Zdobnov EM. BUSCO: assessing genome assembly and annotation completeness with single-copy orthologs. Bioinformatics. 2015;31(19):3210-2. https://doi org/10.1093/bioinformatics/btv351.

16. Beier S, Thiel T, Münch T, Scholz U, Mascher M. MISA-web: a web server for microsatellite prediction. Bioinformatics. 2017;33(16):2583-5. https://doi. org/10.1093/bioinformatics/btx198.

17. Buchfink B, Reuter K, Drost H-G. Sensitive protein alignments at tree-oflife scale using DIAMOND. Nat Methods. 2021;18(4):366-8. https://doi. org/10.1038/s41592-021-01101-X.

18. Cock P, Grüning B, Paszkiewicz K, Pritchard L. Galaxy tools and workflows for sequence analysis with applications in molecular plant pathology. PeerJ. 2013;1(1):e167. https://doi.org/10.7717/peerj.167.

19. Data file 1. Summary and assessment of the data set; 2021). Figshare. https://doi.org/10.6084/m9.figshare.14572125.

20. Data file 2. SSRs of Juniperus squamata; 2021). Figshare. https://doi.org/10. 6084/m9.figshare.14572098.

21. Data file 3. Longest open reading frame prediction; 2021). Figshare. https://doi.org/10.6084/m9.figshare.16870147.

22. Data file 4. Alignment results of Juniperus squamata; 2021). Figshare. https://doi.org/10.6084/m9.figshare.16870333.

23. Data file 5. Go annotation results of Juniperus squamata; 2021). Figshare. https://doi.org/10.6084/m9.figshare.16870401.

24. Data file 6. InterPro analysis results of Juniperus squamata; 2021). Figshare. https://doi.org/10.6084/m9.figshare.16912615.

25. National Center for Biotechnology Information. Sequence reads archive. (2021). https://www.ncbi.nlm.nih.gov/sra/SRR13966305.

26. National Center for Biotechnology Information. Unique transcript isoforms of juniperus squamata. (2021). https://www.ncbi.nlm.nih.gov/sra/ SRR13993906.

27. National Center for Biotechnology Information. Filter unique transcript isoforms for the downstream analysis. (2021). https://www.ncbi.n/m.nih. gov/sra/SRR14000623.

\section{Publisher's Note}

Springer Nature remains neutral with regard to jurisdictional claims in published maps and institutional affiliations.

Ready to submit your research? Choose BMC and benefit from:

- fast, convenient online submission

- thorough peer review by experienced researchers in your field

- rapid publication on acceptance

- support for research data, including large and complex data types

- gold Open Access which fosters wider collaboration and increased citations

- maximum visibility for your research: over 100M website views per year

At BMC, research is always in progress.

Learn more biomedcentral.com/submissions 